\title{
Influence of the Open Kinetic Chain on the Distension of the Transplant after Anterior Cruciate Ligament Surgery with Hamstring Graft: Search for Risk Factors
}

\author{
Morgan Belloir ${ }^{1}$, Jean Mazeas ${ }^{1}$, Maude Traullé ${ }^{1}$, Amaury Vandebrouck ${ }^{2}$, Pascal Duffiet ${ }^{2}$, Louis \\ Ratte $^{2}$ and Florian Forelli ${ }^{1}$ \\ 1 Researcher Physical Therapist, OrthoLab, 85 route de Domont, 95330 Domont, France \\ 2 Knee Orthopaedic Surgeon, Clinic of Domont, 85 route de Domont, 95330 Domont, France
}

\begin{abstract}
Rehabilitation following anterior cruciate ligament reconstruction with hamstring graft allows the patient to regain his functional capacities and to support him in the resumption of sports activities. Rehabilitation also aims to minimize the risk of recurrence, which is why it ensures that the patient's muscular capacities develop properly until they return to sport. Isokinetics helps strengthen and assess the strength of muscle groups in the thigh, but controversy exists as to its use by resistance to the open kinetic chain knee extension that would cause the transplant to distend. The objective of this study is to determine the influence of isokinetic muscle strengthening on the possible laxity of the anterior cruciate ligament and to be able to determine risk factors. The study relates to a population having benefited from anterior cruciate ligament reconstruction with hamstring graft from 3 to 6 months after surgery. Two groups are differentiated, one group exposed to isokinetism during rehabilitation, the other group, named unexposed, undergoes rehabilitation without the use of isokinetism. An anterior knee laxity test is performed 6 months postoperatively using the GNRB ${ }^{\circ}$ machine for all subjects according to the same protocol. The test results were statistically analyzed to determine a relative risk of transplant distension for each group in the study. Comparison of the results of each group by univariate analysis did not reveal any significant result. Multivariate analysis showed interactions in the two strata of the study. It was argued that the use of isokinetics seems to have no effect on the risk of developing distension for the majority of subjects in the exposed group. A tendency towards transplant protection was perceived for each variable except the age under 25 years $(\mathrm{aRR}=1.07)$. The use of isokinetics does not appear to be a cause of transplant distension in patients undergoing an anterior cruciate ligament reconstruction when this method is introduced 3 months postoperatively.
\end{abstract}

Keywords: anterior cruciate ligament; open kinetic chain; isokinetics; laxity

\section{Introduction}

Ligamentization is the natural process that the transplant undergoes after anterior cruciate ligament (ACL) surgery.

This process allows the grafted tendon to obtain the same properties as the previous ligament. Several phases exist in this process, during which the maximum tolerable tension of the transplant varies (1). However, there is no consensus on this phenomenon. The authors' opinions differ in terms of the delays of the phases and the transformations that the graft undergoes (2).

It is for this reason that it is difficult to establish an objective criterion for resumption of sport based on this phenomenon. No scientific evidence validates the time limit as the main criterion for returning to sport (3). 
As part of muscle building, the isokinetic dynamometer provides control over patient position, reinforcement range of motion, speed, mode of contraction, strength developed, intensity and volume of work (4).

The isokinetic dynamometer is commonly used for muscle building. It is a way to build muscle as the patient achieves maximum contraction over the full range (4).

Although a real benefit for muscle rehabilitation, isokinetics is not routinely used during rehabilitation following ACL reconstruction.

In general, the use of opened kinetic chain $(\mathrm{OKC})$ for strengthening the quadriceps is neglected. However, studies highlight its effectiveness in muscle evaluation, but also in its recovery and postoperative rehabilitation (5).

This method remains controversial in particular because of its distal resistance in OKC during the concentric work of the quadriceps, which supposedly have a harmful effect on the transplant.

Today, numerous studies examining the different effects of muscle strengthening rehabilitation in OKC refute this claim $(6,7)$.

Through their work, some authors have concluded that the reinforcement in OKC or in closed kinetic chain (CKC) did not present a significant enough difference to be able to elect one mode superior to the other (6).

The use of OKC is even mentioned as part of a more specific strengthening for a weakened quadriceps (8).

It must be noted that the delays in introducing OKC and the burden of resistance mentioned in the literature do not lead to a consensus.

We can therefore wonder what the influence of OKC is, with isokinetism, on the distension of the transplant after ACL surgery with hamstring graft from 3 months postoperatively.

One can also wonder about the risk factors causing laxity related to the use of OKC.

\section{Materials and Methods}

\subsection{Study Design}

This is a multicentric cohort study using prospective data collection. Subjects were randomly selected from a patient population. The tests were carried out by all participants in the same way to ensure their authenticity, reliability and reproducibility. Study participants were not told about the hypotheses, purpose, and data explored in it. No information on the results was communicated to the patients. The groups underwent two stratifications, one for each study, according to the variables age, body mass index and sex.

A file was submitted to the CNIL with number 920164 . The opinion issued by the Committee for the Protection of Persons (CPP) bears the number 2020-A01115-34. Study participants are informed of the progress of the protocol and the collection of data through an explanatory document serving as informed consent after signature.

\subsection{Participants}

Two groups of subjects participated in the study, one exposed to the use of isokinetics and one unexposed group.

The groups are differentiated by their rehabilitation program following the operation.

The test group, or the exposed group, is composed of subjects who have benefited from rehabilitation associated with muscle strengthening by use of the isokinetic dynamometer with distal resistance applied from the 3rd postoperative month. The control group is composed of subjects who received rehabilitation without the use of an isokinetic dynamometer.

The characteristics of each group are detailed in Table 1.

Table 1. Characteristics of the exposed and unexposed groups (mean \pm standard deviation). 


\begin{tabular}{cccc} 
Age $(\mathbf{y})$ & $29,2 \pm 8,3$ & $31,4 \pm 11,2$ & NS \\
Size (m) & $1,74 \pm 0,1$ & $1,72 \pm 0,1$ & NS \\
Weight (kg) & $74,1 \pm 13,5$ & $71,6 \pm 14,2$ & NS \\
Body Mass Index (kg/m $)$ & $24,4 \pm 3,1$ & $24,2 \pm 3,8$ & NS \\
Sex (M/F) & $23 / 15$ & $23 / 18$ & NS \\
Operated knee (D/G) & $17 / 21$ & $21 / 20$ & NS \\
\hline
\end{tabular}

y: years ; m: meter ; kg: kilogram ; M/F: male/female ; R/L: Right/Left ; NS: Not Significant

The inclusion criteria will be the same for both groups.

The population is made up of human subjects and adults, therefore aged over 18 years.

Subjects must meet the following inclusion criteria: have undergone ACL reconstruction with hamstring graft at 6 months postoperatively. The reconstruction may be isolated or have associated lesions. The exclusion criteria are the same for both groups.

At the time of assessment, there should be no signs of inflammation, the presence of postoperative lesions on one of the knees, knee pain (Analog Visual Scale $<20$ ).

\subsection{Protocol}

All the subjects of the two groups are tested by the same examiner and following the same protocol, 3 thrusts at 250 Newtons $(\mathrm{N})$. The measurement acquisition time is 10 minutes. Data were collected between September 2019 and March 2020. Testing occurred 6.6 ( \pm 1.4) months postoperatively for the exposed group and $6.4( \pm 1.1)$ months for the non-group. exposed.

\subsection{Material}

The measurements were carried out using the GNRB® robotic laximeter. Its reproducibility was judged to be good, with an intraclass correlation coefficient measured at 0.90 for 134 and $250 \mathrm{~N}$. We chose the threshold value of $134 \mathrm{~N}$ which has a sensitivity of $70 \%$ and a specificity of $99 \%$ (9). A differential greater than or equal to $3 \mathrm{~mm}$ at $134 \mathrm{~N}$ between the two knees is considered as ACL distension. A differential less than $1 \mathrm{~mm}$ at $134 \mathrm{~N}$ is judged to be anatomically comparable.

The data used for this study are the differentials of the translations at $134 \mathrm{~N}$ of each subject, as well as their physical characteristics.

The GNRB ${ }^{\circledR}$ is connected to a DELL $^{\circledR}$ computer. This computer has a 256 GB SSD, a 1 TB hard drive, an Intel Core $i 7^{\circledR}$ processor, 8 GB of RAM, and Windows $10^{\circledR}$. GNRB ${ }^{\circledR}$ software, (version 1.4.3 FR), is installed on this device.

\subsection{Statistical analysis}

Statistical analysis was performed using $R^{\circledR}$ software (R Studio, Version 1.2.5033 ${ }^{\circ}$ 2009- 2019 RStudio, Inc - 250 Northern Ave, Boston, MA 02210) and Excel ${ }^{\circledR}$ as a first step.

As the study consisted of 79 subjects, verification of the normality of the distributions of each group was carried out by a Shapiro test. Then, in order to determine the homogeneity of the population, a Pearson Chi Square test is carried out for the qualitative variables (sex, injured side) and a Mann-Whitney for the quantitative variables (age, height, weight, BMI).

Secondly, interference statistics are established with the results of the laximetric test of each subject. They are performed using Epi InfoTM I CDC software.

The confidence interval (CI) is established for CI $=95 \%$ and the level of significance $\alpha=0.05$.

This involves establishing a relative risk by univariate analysis for the results of each group. This, in order to compare the risk of distension of the exposed and non-exposed groups. The inverse ratio is also calculated. 
These calculated relative risks correspond to the relative risks (RR) of this study. The significance of these results is determined by Fisher's exact test.

Each stratification undergoes univariate analysis to derive an adjusted relative risk (aRR). These results will make it possible to deduce whether the variable influences the occurrence of distension.

The significance of the analysis is also calculated by Fisher's exact test.

Then, a multivariate analysis is performed by the Cochran-Mantel-Haenszel (MH) adjustment method for each stratification, in order to eliminate any confounding factors.

Confounding factors are also removed by multivariate analysis using the $\mathrm{CMH}$ adjustment method.

\section{Results}

No statistically significant difference was detected between the control group and the test group in the characteristics of age, height, weight, Body Mass Index (BMI) and sex ( $\rho$-value>0.05).

The variances are said to be identical, the distribution of the population is normal.

Table 2. Result of the exposed and non-exposed groups following the test with calculation of the RR for a differential at $3 \mathrm{~mm}$.

\begin{tabular}{|c|c|c|c|c|c|c|c|}
\hline & \multirow{2}{*}{\multicolumn{2}{|c|}{$\begin{array}{l}\Delta \geq 3(\mathrm{~mm}) \\
(\mathrm{n}=4)\end{array}$}} & \multicolumn{2}{|c|}{$\Delta<3(\mathrm{~mm})$} & \multirow[b]{2}{*}{$\mathbf{R R}$} & \multirow[b]{2}{*}{ CI 95\% } & \multirow[b]{2}{*}{$\rho$} \\
\hline & & & $(n=75)$ & $(\%)$ & & & \\
\hline Exposed & 2 & $(5,3)$ & 36 & $\begin{array}{c}(94,7 \\
)\end{array}$ & 1,08 & {$[0,16-7,28]$} & NS \\
\hline Unexposed & 2 & $(4,9)$ & 39 & $\begin{array}{c}(95,1 \\
)\end{array}$ & 0,93 & {$[0,14-6,26]$} & NS \\
\hline \multicolumn{8}{|c|}{$\begin{array}{c}\text { NS = Not Significant } \\
\text { RR }=\text { Risk Relativ } \\
\mathrm{Cl}=\text { Confidence Interval } \\
=\text { Differential at } 134 \mathrm{~N} \text { at GNRB }{ }^{\circledR}\end{array}$} \\
\hline
\end{tabular}

Univariate analysis shows no statistically significant difference for each of the groups. The RR established for the exposed group is 1.08 , it is 0.93 for the unexposed group.

Table 3. Result of multivariate analysis of the exposed group for a differential at $3 \mathrm{~mm}$.

\begin{tabular}{cccccc}
\hline$\Delta \geq 3 \mathrm{~mm}$ & $\Delta<3 \mathrm{~mm}$ & $a R R$ & $C I 95 \%$ & $\rho(M H)$ & $\rho$ \\
\cline { 1 - 5 }$(\mathrm{n}=2)$ & $(\mathrm{n}=36)$ & & & \\
\hline
\end{tabular}




\section{Age (years)}

\begin{tabular}{|c|c|c|c|c|c|c|c|}
\hline $18-25 y$ & 1 & $(2,6)$ & 13 & $(34,2)$ & 1,07 & {$[0,07-15,54]$} & NS \\
\hline $26-35 y$ & 1 & $(2,6)$ & 13 & $(34,2)$ & 0,86 & {$[0,06-12,28]$} & 0 \\
\hline$>35 y$ & $\varnothing$ & $(0)$ & 10 & $(26,3)$ & -1 & & NS \\
\hline
\end{tabular}

\section{BMI $\left(\mathrm{kg} / \mathrm{m}^{2}\right)$}

\begin{tabular}{|c|c|c|c|c|c|c|c|}
\hline $18,5-24,9$ & 1 & $(2,6)$ & 21 & $(55,3)$ & 1,14 & {$[0,07-17,11]$} & NS \\
\hline$\geq 25$ & 1 & $(2,6)$ & 15 & $(39,5)$ & -1 & & NS \\
\hline
\end{tabular}

\section{Sex}

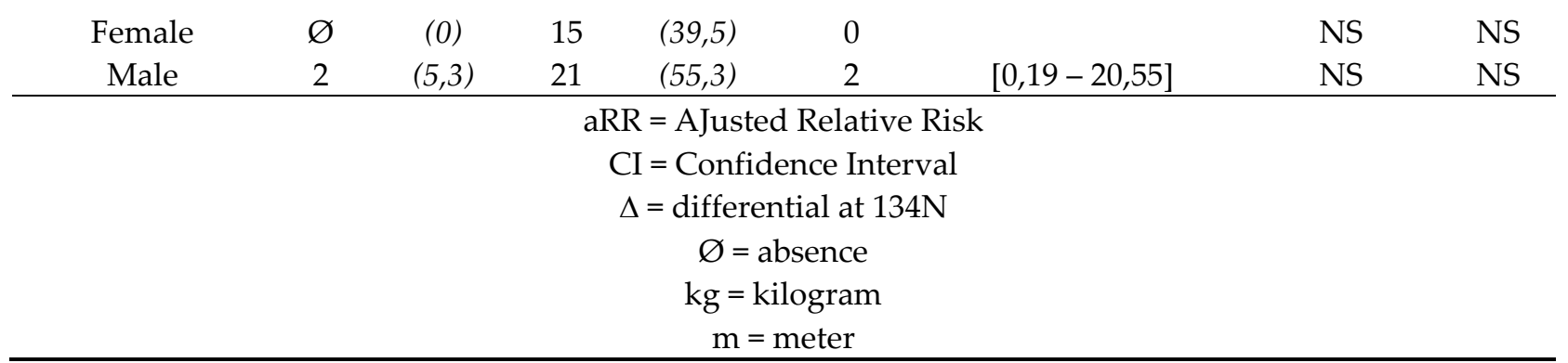

Across all the variables where the statistical analysis does not show a significant difference, there is a link between the risk and the age variable between 26 and 35 years old. Multivariate statistical analysis highlights a statistically significant difference for this variable.

Table 4. Result of the exposed and non-exposed groups following the test with calculation of the RRg for a differential at $1 \mathrm{~mm}$.

\begin{tabular}{|c|c|c|c|c|c|c|c|}
\hline & \multicolumn{2}{|c|}{$\Delta>1 \mathrm{~mm}$} & \multicolumn{2}{|c|}{$\Delta \leq 1 \mathrm{~mm}$} & \multirow[b]{2}{*}{$\mathbf{R R}$} & \multirow[b]{2}{*}{ CI 95\% } & \multirow[b]{2}{*}{$\rho$} \\
\hline & $(n=39)$ & & $(n=40)$ & & & & \\
\hline Exposed & 15 & $(39,5)$ & 23 & $(60,5)$ & 0,67 & {$[0,42-1,08]$} & NS \\
\hline Unexposed & 24 & $(58,5)$ & 17 & $(41,5)$ & 1,48 & {$[0,93-2,37]$} & NS \\
\hline \multicolumn{8}{|c|}{$\begin{array}{l}\text { NS = non significant } \\
\text { RR = Relative Risk } \\
I=\text { Confidence Interv }\end{array}$} \\
\hline
\end{tabular}

Table 5. Result of the multivariate analysis of the exposed group for a differential at $1 \mathrm{~mm}$.

\begin{tabular}{ccccccc}
\hline$\Delta>1 \mathrm{~mm}$ & $\Delta \leq 1 \mathrm{~mm}$ & $a R R$ & $C I 95 \%$ & $\rho(\mathrm{MH})$ & $\rho$ \\
\cline { 2 - 3 } & $(\mathrm{n}=15)$ & $(\mathrm{n}=23)$ & & & &
\end{tabular}




\section{Age (years)}

$\begin{array}{ccccccccc}18-25 y & 7 & (18,4) & 7 & (18,4) & 1,07 & {[0,50-2,27]} & \text { NS } & \text { NS } \\ 26-35 \text { y } & 5 & (13,2) & 9 & (23,7) & 0,48 & {[0,22-1,03]} & 0,01 & \text { NS } \\ >35 y & 3 & (7,9) & 7 & (18,4) & 0,53 & {[0,18-1,5]} & 0,04 & \text { NS }\end{array}$

\section{BMI $\left(\mathrm{kg} / \mathrm{m}^{2}\right)$}

$\begin{array}{ccccccccc}18,5-24,9 & 10 & (26,3) & 12 & (31,6) & 0,87 & {[0,48-1,58]} & \text { NS } & \text { NS } \\ \geq 25 & 5 & (13,2) & 11 & (28,9) & 0,52 & {[0,23-1,20]} & 0,02 & \text { NS }\end{array}$

Sex

\begin{tabular}{ccccccccc} 
Female & 4 & $(10,5)$ & 11 & $(28,9)$ & 0,53 & {$[0,20-1,39]$} & NS & NS \\
Male & 11 & $(28,9)$ & 12 & $(31,6)$ & 0,73 & {$[0,44-1,23]$} & 0,04 & NS \\
\hline
\end{tabular}

$\mathrm{aRR}=$ AJusted Relative Risk

$\mathrm{CI}=$ Confidence Interval

$\Delta=$ differential at $134 \mathrm{~N}$

$\mathrm{kg}=$ kilogram

$\mathrm{m}=$ meter

The analysis does not show any statistically significant difference for each of the groups. The RR established for the group with isokinetics is 0.67 , it is 1.48 for the group without isokinetics. (Table 4).

Across all the variables where the statistical analysis does not show a statistically significant difference, there is a link between the risk and the variables age over 25 years, BMI over $25 \mathrm{~kg} . \mathrm{m}^{2}{ }^{2}$ and male sex. Multivariate statistical analysis allows the demonstration of a significant difference for these variables. (Table 5)

\section{Discussion}

The controversy in ACL reconstruction is that the use of isokinetics applied to the knee is OKC with distal resistance for quadriceps work.

Indeed, this resisted extension movement associates an anterior sliding of the tibia on the femur, which is normally limited by the tensioning of the ACL (10).

Stresses exerted in this way on an ACL transplant shortly after surgery would be harmful to it because of its reduced mechanical strength. It risks elongation and therefore laxity of the joint or even rupture in the worst case (11).

This is why extension work in resisted OKC seems contraindicated in the first weeks after surgery.

However, some authors highlight the benefit of using OKC postoperatively for muscle strengthening, but also for the phenomenon of transplant ligamentization. However, this way of working is not necessarily widespread (12).

The interest of this study is to test the influence of muscle strengthening in OKC with distal resistance using the isokinetic dynamometer, from 12 post-operative weeks up to 6 months after ACL surgery with hamstring graft on the possible laxity of the transplant.

Numerous studies have focused on the contributions and consequences of OKC with distal resistance after ACL reconstruction at different times during rehabilitation.

Long accepted as deleterious for the transplant in the first weeks of rehabilitation, recent literature has refuted many beliefs on this subject, agreeing with the results obtained.

This is the case for the study by Fukuda et al (7) which focused on the early use of OKC reinforcement. Their results have reinforced the findings of this study. 
In their study, they compared the laximetry results of different groups of a population who had received ACL reconstruction with hamstring graft. The groups differed in their time to introduce resistance strengthening of the quadriceps in OKC as well as by the authorized range of work. Reinforcement had started in the 4th postoperative week for the first group and at the 12th for the second. The study lasted for 25 weeks with 3 strengthening sessions per week.

Anterior knee laxity was assessed following the strengthening program for each group. No statistically significant difference was found in the results of the laxity tests, it was concluded from the results of this study that an early start at 4 weeks of reinforcement with OKC does not differ from a late onset at 12 weeks in terms of repercussion on the anterior laxity of the knee.

Nevertheless, the meta-analysis by Perriman et al (13), on the repercussions of OKC and CKC on transplant laxity did not conclude on a real interest in the early introduction of OKC.

It was retained that the effects of using OKC introduced early in rehabilitation vary depending on the site of the graft harvest. In fact, in the different studies used, the ACL reconstructions with Kenneth-Jones showed no significant difference in laxity, for a strengthening of the quadriceps by OKC introduced at the 3rd postoperative week in certain cases.

Contrary to our results, laxity was detected in some studies in subjects who received reconstruction with hamstring graft. Too early resistance and too rapid full extension of the knee were cited as the cause of this laxity.

During the same period studied, a work between 60 and $90^{\circ}$ of flexion would have been more suitable because of the minimal stresses applied to the graft in this amplitude.

It was concluded that the evidence was limited for an early introduction of OKC in terms of anterior tibial laxity and knee function.

Our results agree with the study by Heijne et al (14). Their objective was to assess the outcome of knee function in terms of anterior tibial laxity after early initiation of OKC. The population consisted of subjects who had ACL reconstruction with hamstring graft for one group and with Kenneth Jones for another. For each group, two subgroups were differentiated by their postoperative times of introduction of work in OKC.

The strengthening of the quadriceps in OKC began at the 4th postoperative week for the first subgroup and at the 12th for the second.

The resistance force was progressive during the study, it changed according to the subject's tolerance. Assessments at 3, 5 and 7 months postoperatively were performed for each group.

The results showed a significantly higher mean anterior tibial translation difference of $1.0 \mathrm{~mm}$ for the group operated with hamstring graft with early introduction of OKC compared to the group operated on by Kenneth-Jones.

A statistically significant difference was detected between the results of the hamstring graft groups, with a higher mean anterior translation of $1.2 \mathrm{~mm}$ for the group with early introduction.

The study therefore concluded that the early introduction of OKC resulted in greater anterior translation for ACL reconstruction with hamstring graft compared to one with Kenneth-Jones. However, the results obtained in the hamstring graft group, as in our study, do not show a differential greater than $3 \mathrm{~mm}$.

The study by Perry et al (15) showed that the effects of OKC and CKC do not have any statistically significant difference on graft laxity midway through rehabilitation.

The study involved two groups who received ACL reconstruction. One group had carried out a strengthening program with OKC the other with CKC. Both groups performed this muscle strengthening between the 8 th and 14 th postoperative week.

It is specified that no other resistance exercise has been introduced in the rehabilitation of knee muscle strength during this period.

Our results are therefore comparable to those of this study as far as the populations, study times and cohort method converge.

The work of Morissey et al (16) also refuted the belief of a transplant distension effect of reinforcement in OKC against resistance in the quadriceps, as previously demonstrated, our results agree with this study. 
Their work supported the interest in postoperative transplant tensioning. The constraining action on the transplant would reduce laxity by stimulating the production of collagen.

They also reinforced the idea that the same stresses were placed on the ACL during exercise in $\mathrm{OKC}$ and $\mathrm{CKC}$, while recognizing that workload obviously affects the stress caused.

This study shows a beneficial effect on the use of OKC as part of rehabilitation after ACL reconstruction.

The study by Bieler et al (17) sought to show the value of high intensity postoperative rehabilitation compared to low intensity in muscle strengthening. The study was carried out from the 8th to the 20th postoperative week in a population ranging from 18 to 45 years old.

She found that resistance training through a variety of exercises, including high intensity resistance OKC knee extension during rehabilitation, significantly improved extensor muscle power and knee function. No consequences on joint laxity were observed during end-of-protocol tests.

Our results are consistent with the conclusions of this study. Study timelines also coincide even though their protocol introduced OKC previously.

Reinforcement by the use of resistance OKC with intense contraction of the quadriceps has no effect on possible anterior laxity of the knee.

The literature also agrees that strengthening the muscles of the thigh muscles reduces anterior knee laxity $(18,19)$

Also, a study by Barcellona et al (18) showed the results of an OKC knee strengthening program.

Eighteen subjects aged between 18 and 60 underwent, following an ACL injury, standardized rehabilitation, coupled with a rehabilitation program for twelve weeks. The program itself was a OKC strengthening of the quadriceps of two sets of twenty repetitions at maximum load. The results showed an improvement in anterior laxity compared to conventional rehabilitation. It was deduced that strengthening the thigh muscle would reduce knee laxity.

The study by Mikkelsen et al (20) compared two methods of muscle strengthening after ACL surgery. Our results are consistent with this study.

The two methods differed in muscle strengthening, one used only CKC exercises while the other combined CKC and OKC from the 6th postoperative week. The sample consisted of subjects between 19 and 40 years of age.

Laximetry tests at 6 months postoperatively showed no significant difference in laxity between the two groups. In addition, a significantly higher quadriceps strength was noted for the subjects of the group combining CKC and OKC during muscle strengthening.

A larger number of subjects $(n=12)$ had returned to their sports activities at the same level in the group combining CKC and OKC ( $\mathrm{n}=5$ for the CKC group). Their returns were made on average 2 months earlier than those of the subjects of the CKC group.

This study supports the results obtained, it also highlights the benefits of using OKC on quadriceps strength. It would be interesting to study the recidivism rate in those subjects who returned to sports activities earlier.

A study conducted by Crisitiani et al (21) on a population of 5,462 subjects sought to identify risk factors for distension of the graft after surgical reconstruction of the ACL.

Tests for the anterior laxity of the knee were done before the operation and six months after it.

The results obtained in our study do not agree with the conclusions of this study, since it identifies the age of less than 30 years as a risk factor for laxity after ACL reconstruction.

A trend with no effect on ACL distension was found for this variable when it is associated with a strengthening of the quadriceps in OKC after analysis of our results obtained.

It is possible that the origin of these opposing findings is the small number of subjects composing our sample compared to that of Cristiani et al.

It also identified the hamstring graft as a factor in laxity after ACL reconstruction. For our part, having only had patients operated with hamstring graft and a low rate of laxity in the whole population, we can think that a difference in surgical or rehabilitation management influences these results.

A study by Liu et al (12) supported the results of the study. The aim was to study the effects of isokinetic strengthening of the thigh muscle groups on graft remodeling. A protocol was set up for a 
control group and a group having used isokinetics 3 to 6 months postoperatively. Magnetic resonance imaging of the graft was followed in order to follow the evolution of the shape, the tension and the degree of vascularization at 3, 6 and 12 months postoperatively. Second-look arthroscopy was performed approximately 23 months after ACL reconstruction.

Significantly better torque and hamstring-to-quadriceps ratio were found in the isokinetics group when tested at 6 and 12 months. The graft was judged to be of significantly better quality at 12 months for the group with isokinetics. A higher histological score was found for this group during the second arthroscopy.

It was concluded that the use of isokinetics would allow early recovery of muscle strength as well as early remodeling of the graft. However, the analyzes carried out in the present study do not make it possible to highlight the ligamentization parameters mentioned above. An additional study including the two protocols would be interesting to reveal results in line with the studies cited.

The systematic review by Janssen et al (22) focused on the clinical results of accelerated rehabilitation after ACL reconstruction with hamstring graft.

Her review included 24 different studies, she concluded, among other things, that the onset of OKC from the 4th postoperative week in a limited range of motion between 90 and $45^{\circ}$ flexion did not result in lengthening of the transplant.

This study joins the literature that evokes the early introduction of work in OKC. Although our study did not focus on a specific area of work, our results seem to agree with the conclusions of Janssen et al regarding the repercussions on reconstrcution surgery.

In this sense, the systematic review by Van Grivsen et al (23) joins that of Janssen et al (22) mentioned previously regarding the introduction of OKC in rehabilitation. Their review showed that the use of OKC for muscle building over an amplitude between 90 and $40^{\circ}$ of flexion significantly increases quadriceps strength without causing transplant laxity. This introduction was mentioned early between the 2 nd and the 9 th post-operative week, in order to prevent the quadriceps muscular atrophy from continuing.

Our results are in agreement with the latter, showing that the strengthening of the quadriceps OKC muscle soon after the operation is not detrimental to the transplant. They also discussed the long-term benefits of using $\mathrm{OKC}$ for the quadriceps.

\section{Limits}

On the one hand, the small sample of the study is a first limit to the results obtained. Some studies found in the literature have a significantly higher number of participants, which is why it is difficult to generalize the results obtained as well as to compare them.

On the other hand, the present study looked at a population who had been operated on for ACL reconstruction using a single technique. Indeed, all the subjects having been operated on hamstring graft, one can think that the results only apply to people who have undergone surgery by this technique.

A new stratum concerning other surgical techniques would be necessary to account for the action of the use of isokinetics on the graft, with the possibility of introducing OKC even earlier in rehabilitation.

An additional study focusing on the analysis of the different variables of each stratum would also be beneficial to deepen the search for risk factors. Our study is interested in the interactions between the use of isokinetics and the appearance of distension for the selected variables, but it does not take into account the interactions of the variables between them.

\section{Conclusion}

The main objective of the study was to determine whether strengthening quadriceps muscle in $\mathrm{OKC}$ with isokinetics at 3 months postoperatively would cause transplant distension.

The study set out to identify risk factors for distension associated with the use of isokinetics.

To do so, the study followed a cohort model between a group exposed to isokinetism from the 3rd postoperative month and an unexposed group. Anterior tibial translation measurement 
differentials were evaluated with the use of the GNRB®. A differential greater than $3 \mathrm{~mm}$ measured at $134 \mathrm{~N}$ was considered to be transplant laxity. Statistical analysis of the differential results of each of the groups did not show any significant difference, and did not appear to show any effects for the exposed subjects on possible distension of the transplant. Nevertheless, a tendency towards protection for the risk of distension is found in subjects aged between 26 and 35 years of the exposed group. Although the result obtained was not significant, a tendency towards protection was found for the subjects of the exposed group with regard to an alteration in quality leading to an anatomically comparable difference (differential $>1 \mathrm{~mm}$ ). The use of isokinetic work in the context of reinforcement is indicated as beneficial in the literature. By its computerized control, it allows a follow-up, session after session, of the muscular performances. It informs of the existence of a muscular imbalance between the quadriceps and the hamstrings, and therefore leaves the possibility of adapting the following sessions in order to rebalance the forces in the presence of the knee.

The possibility of developing an evolving program according to the speed, the mode of contraction or the range of motion offers a significant choice of strengthening methods for the physiotherapist in the rehabilitation of muscle function after an ACL reconstruction.

Finally, the maximum muscle contraction the patient makes over the full range of motion makes isokinetics a great way to strengthen muscle function.

Conflicts of Interest: The authors declare that they have no conflict of interest

Funding: This research and its authors have received no funding

\section{References}

1. Scheffler SU, Unterhauser FN, Weiler A. Graft remodeling and ligamentization after cruciate ligament reconstruction. Knee Surg Sports Traumatol Arthrosc. sept 2008;16(9):834-42.

2. Claes S, Verdonk P, Forsyth R, Bellemans J. The "Ligamentization" Process in Anterior Cruciate Ligament Reconstruction: What Happens to the Human Graft? A Systematic Review of the Literature. Am J Sports Med. nov 2011;39(11):2476-83.

3. Burgi CR, Peters S, Ardern CL, Magill JR, Gomez CD, Sylvain J, et al. Which criteria are used to clear patients to return to sport after primary ACL reconstruction? A scoping review. Br J Sports Med. sept 2019;53(18):1154-61.

4. Edouard, P., Degache, F. Rééducation: Intérêt de l'isocinétisme. In: Guide d'isocinétisme. Elsevier Masson; 2016.

5. Cvjetkovic D, Bijeljac S, Palija S, Talic G, Radulovic T, Kosanovic M, et al. Isokinetic Testing in Evaluation Rehabilitation Outcome After ACL Reconstruction. Med Arch. 2015;69(1):21.

6. Jewiss D, Ostman C, Smart N. Open versus Closed Kinetic Chain Exercises following an Anterior Cruciate Ligament Reconstruction: A Systematic Review and Meta-Analysis. J Sports Med.

7. Fukuda TY, Fingerhut D, Moreira VC, Camarini PMF, Scodeller NF, Duarte A, et al. Open Kinetic Chain Exercises in a Restricted Range of Motion After Anterior Cruciate Ligament Reconstruction: A Randomized Controlled Clinical Trial. Am J Sports Med. avr 2013;41(4):788-94.

8. Mc Nair, P. Force musculaire et rééducation proprioceptive du genou après ligamentoplastie du croisé antérieur. Kinésithérapie Rev. févr 2009;9(85-86):49.

9. Robert H, Nouveau S, Gageot S, Gagnière B. A new knee arthrometer, the GNRB®: Experience in ACL complete and partial tears. Orthop Traumatol Surg Res. mai 2009;95(3):171-6.

10. Christel P. Ligament croisé antérieur et stabilité rotatoire. Rev Chir Orthopédique Réparatrice Appar Mot. déc 2005;91(8):18-22.

11. Nicholettos A, Barcellona MG, Morrissey MC. The immediate effects of open kinetic chain knee extensor exercise at different loads on knee anterior laxity in the uninjured. The Knee. déc 2013;20(6):500-4.

12. Liu H, Lu W, Liang D, Geng H, Zhu W, Ouyang K, et al. [Effect of isokinetic training of thigh muscle group on graft remodeling after anterior cruciate ligament reconstruction]. Chin J Reconstr Surg. sept 2019;33(9):1088-94.

13. Perriman A, Leahy E, Semciw AI. The Effect of Open- Versus Closed-Kinetic-Chain Exercises on Anterior Tibial Laxity, Strength, and Function Following Anterior Cruciate Ligament Reconstruction: A Systematic Review and Meta-analysis. J Orthop Sports Phys Ther. juill 2018;48(7):552-66.

14. Heijne A, Werner S. Early versus late start of open kinetic chain quadriceps exercises after ACL reconstruction with patellar tendon or hamstring grafts: a prospective randomized outcome study. Knee Surg Sports Traumatol Arthrosc. 2 avr 2007;15(4):402-14. 
15. Perry MC, Morrissey MC, King JB, Morrissey D, Earnshaw P. Effects of closed versus open kinetic chain knee extensor resistance training on knee laxity and leg function in patients during the 8- to 14-week postoperative period after anterior cruciate ligament reconstruction. Knee Surg Sports Traumatol Arthrosc. juill 2005;13(5):357-69.

16. Morrissey MC, Perry MC, King JB. Is Knee Laxity Change After ACL Injury and Surgery Related to Open Kinetic Chain Knee Extensor Training Load?: Am J Phys Med Rehabil. mai 2009;88(5):369-75.

17. Bieler T, Aue Sobol N, Andersen LL, Kiel P, Løfholm P, Aagaard P, et al. The Effects of High-Intensity versus Low-Intensity Resistance Training on Leg Extensor Power and Recovery of Knee Function after ACLReconstruction. BioMed Res Int. 2014;2014:1-11.

18. Barcellona MG, Morrissey MC, Milligan P, Clinton M, Amis AA. The effect of knee extensor open kinetic chain resistance training in the ACL-injured knee. Knee Surg Sports Traumatol Arthrosc. nov 2015;23(11):3168-77.

19. Palmieri-Smith RM, Strickland M, Lepley LK. Hamstring Muscle Activity After Primary Anterior Cruciate Ligament Reconstruction-A Protective Mechanism in Those Who Do Not Sustain a Secondary Injury? A Preliminary Study. Sports Health Multidiscip Approach. juill 2019;11(4):316-23.

20. Mikkelsen C, Werner S, Eriksson E. Closed kinetic chain alone compared to combined open and closed kinetic chain exercises for quadriceps strengthening after anterior cruciate ligament reconstruction with respect to return to sports: a prospective matched follow-up study. Knee Surg Sports Traumatol Arthrosc. nov 2000;8(6):337-42.

21. Cristiani R, Forssblad M, Engström B, Edman G, Stålman A. Risk Factors for Abnormal Anteroposterior Knee Laxity After Primary Anterior Cruciate Ligament Reconstruction. Arthrosc J Arthrosc Relat Surg. août 2018;34(8):2478-84.

22. Janssen RPA, van Melick N, van Mourik JBA, Reijman M, van Rhijn LW. ACL reconstruction with hamstring tendon autograft and accelerated brace-free rehabilitation: a systematic review of clinical outcomes. BMJ Open Sport Exerc Med. avr 2018;4(1):e000301.

23. Van Grinsven S, van Cingel REH, Holla CJM, van Loon CJM. Evidence-based rehabilitation following anterior cruciate ligament reconstruction. Knee Surg Sports Traumatol Arthrosc. août 2010;18(8):1128-44

(C) 2020 by the authors. Submitted for possible open access publication under the terms and conditions of the Creative Commons Attribution (CC BY) license (http://creativecommons.org/licenses/by/4.0/). 